\title{
Acknowledgement to Reviewers of Life in 2019
}

\section{Life Editorial Office}

MDPI, St. Alban-Anlage 66, 4052 Basel, Switzerland

Published: 17 January 2020

The editorial team greatly appreciates the reviewers who have dedicated their considerable time and expertise to the journal's rigorous editorial process over the past 12 months, regardless of whether the papers are finally published or not. In 2019, a total of 78 papers were published in the journal, with a median time to first decision of 15 days and a median time from submission to publication of 47 days. The editors would like to express their sincere gratitude to the following reviewers for their generous contribution in 2019:

Adam, Zachary

Albornoz, Francisco

Amils, Ricardo

Amundson, Ronald

Bains, William

Baqué, Mickael

Bartlett, Stuart

Basak, Shibaji

Baum, David A.

Bejaoui, Salma

Bernhardt, Harold S.

Bonfanti, Luca

Borda, Michael

Brack, Andre

Brack, André

Braun, Dieter

Broman, Elias

Buhse, Thomas

Burton, Aaron S.

Burton, Zachary $\mathrm{F}$

Burton, Zachary F.

Cafferty, Brian

Callejón-Ferre, Ángel-Jesús

Chan, Clement

Chatzitheodoridis, Elias

Clarke, Paul

Claudi, Riccardo

Corenblit, Dov

Cronin, Leroy

Czárán, Tamás

Danger, Grégoire

Davank, Vadim

De La Escosura, Andrés

De Los Santos, Emmanuel Lorenzo

\author{
Deamer, David \\ Dhakal, Dipesh \\ Di Giulio, Massimo \\ Do, Chi-Wai \\ Dołowy, Krzysztof \\ Doolittle, Russell F. \\ Dreamer, David \\ Duvernay, Fabrice \\ Egel, Richard \\ Erastova, Valentina \\ Fahrenbach, Albert \\ Ferrante, Antonio \\ Finster, Kai \\ Fiore, Michele \\ Fologea, Daniel \\ Fornaro, Teresa \\ Forsythe, Jay \\ Frenkel-Pinter, Moran \\ Frossard, Jean-Pierre \\ Fullmer, Matthew \\ Furukawa, Yoshihiro \\ Gagnon, Jean-Sebastien \\ Garner, Charles \\ Georgelin, Thomas \\ Giner-Lamia, Joaquin \\ Grothe, Hinrich \\ Grover, Martha A \\ Hao, Jihua \\ Heckl, Dirk \\ Hendrix, Donald A. \\ Hickman-Lewis, Keyron \\ Higgs, Paul \\ Hitzeroth, Inga \\ Horváth, Dezsö
}


Horváth, Judit

Hsing, Yue-Ie

Huertas Romera, María José

Ichihashi, Norikazu

Ichihashi, Norikazu

Ikehara, Kenji

Jafarpour, Farshid

Jakubowski, Hieronim

Jheeta, Sohan

Jia, Tony

Jones, Davey

Jordan, Sean

Judit, Šponer

Kaddour, Hussein

Kakegawa, Takeshi

Karunakaran, Suneesh

Katin, Konstantin P.

Kawasaki, Tsuneomi

Kee, Terence

Keinath, Melissa

Kieft, Tom

Kippes, Nestor

Kitadai, Norio

Kobayashi, Kensei

Könnyü, Balázs

Kubyshkin, Vladimir

Kun, Ádám

Kunnev, Dimiter

Lambert, Jean-Francois

Lan, Ethan

Lancet, Doron

Lascelles, Desmond

Lezcano Vega, María Ángeles

Li, Yamei

Lingam, Manasvi

Lopez Maury, Luis

$\mathrm{Lu}$, You

Lundin, Daniel

Magnuson, Ann

Malinge, Jean-Marc

Mansy, Sheref

Markovitch, Omer

Martínez-Romero, Esperanza

Mascetti, Joëlle

Matsuura, Tomoaki

Mátyás, Bence

Mauksch, Michael

Maurel, Marie-Christine

Maurel, Marie-Christine

Michaelian, Karo

Mizuuchi, Ryo

Moran, Joseph
Muchowska, Kamila B

Murugaiyan, Jayaseelan

Nanda, Sitansu Sekhar

Naraoka, Hiroshi

Newman, Stuart A.

Nghe, Philippe

Noffke, Nora

Novikov, Andriy

Nuevo, Michel

Park, Thomas

Peacock-López, Enrique

Pedreira-Segade, Ulysse

Pinheiro, Vitor

Plasson, Raphael

Polyansky, Anton A.

Popova, Alexandra A.

Potter-McIntyre, Sally L.

Potvin-Trottier, Laurent

Powner, Matthew

Preiner, Martina

Rabbow, Elke

Rajamani, Sudha

Ralser, Markus

Ramirez, Ramses

Ramirez, Ramses

Ribó, Josep M.

Riek, Roland

Rimmer, Paul

Rimmer, Paul

Roberts, Irene

Rosi, Marzio

Ross, David S.

Rossi, Jean-Christophe

Rovner, Alexis

Ruiz-Mirazo, Kepa

Saad, Nizar Y.

Saha, Ranajay

Sahai, Nita

Sakamoto, Kensaku

Seligmann, Hervé

Shcherbakova, Viktoria

Shiomi, Daisuke

Shirt-Ediss, Ben

Silvano, Onofri

Smith, Eric

Solovchenko, Alexei

Soumillion, Patrice

Speijer, Dave

Speth, Daan R.

Sponer, Judit

Stano, Pasquale

Stern, Jennifer C. 
Stevens, Adam H.

Strasdeit, Henry

Strazewski, Peter

Strazewski, Peter

Succurro, Antonella

Surman, Andrew

Szabo, Erik

Szathmáry, Eörs

Takeuchi, Nobuto

Tamarit, Daniel

Tamura, Koji

Taubner, Ruth-Sophie

Taylor, Annette

Tessera, Marc

Tkachenko, Alexei

Toner, Jonathan

Torres, Adrian Gabriel

Trindle, Carl
Vallée, Yannick

Van Delden, Sander

Vasconcelos, F. A.

Von Der Haar, Tobias

Wagner, Nathaniel

Wang, Anna

Wang, Anna

Weigand, Wolfgang

Wills, Peter R.

Yabuta, Hikaru

Yang, Jyisy

Yavin, Eylon

Yin, John

Zagrovic, Bojan

Zanghellini, Jürgen

Zobel, Patrick

Zrzavá, Magda

Zuo, Ran

(c) 2020 by the author. Licensee MDPI, Basel, Switzerland. This article is an open access article distributed under the terms and conditions of the Creative Commons Attribution (CC BY) license (http://creativecommons.org/licenses/by/4.0/). 